\title{
Das Gesicht der Aufsteiger: Römische Freigelassene und die Ideologie der Elite ${ }^{*}$
}

\author{
BARBARA BORG (HEIDELBERG)
}

Wohl kaum eine antike Kunstgattung hat eine ähnlich zwiespältige Rezeption erfahren wie die römischen Porträts. Auf der einen Seite schien die lebensnahe Darstellung der Köpfe von runzligen Greisen, heldenhaften Militärs, stutzerhaften Jünglingen, schönen, eleganten Frauen und niedlichen Mädchen diese Bildnisse geradezu zum Inbegriff mimetischer Kunst zu bestimmen. Dadurch waren sie schon seit Winckelmann für die Mehrzahl der Kunstkenner und -theoretiker von vornherein in eine niedere Klasse verwiesen oder gar gänzlich aus dem Bereich wahrer Kunst verbannt. Aber auch als sich im 19. Jh. die Ansicht durchzusetzen begann, daß nicht nur „Kunstwerke“ im engeren Sinne, sondern die gesamte materielle Hinterlassenschaft der Antike bedeutsam sei, da das Erkenntnisziel nun zunehmend ein historisches wurde, schienen die Porträts der Römer eben wegen ihrer scheinbar fotografischen, nur die physische Realität widerspiegelnden Darstellungsweise zu diesem Ziel wenig beizutragen.

Auf der anderen Seite belegt nicht zuletzt die Sammelpraxis ein seit der Renaissance anhaltendes Interesse an solchen Porträts, die gelegentlich zu Galerien der ,guten Herrscher“, antiker Philosophen, Dichter usw. zusammengestellt und notfalls durch moderne Büsten ergänzt wurden, oftmals aber auch allein nach Gelegenheit gesammelt und präsentiert wurden. In beiden Fällen scheint ein oftmals nur diffuses Gefühl eine Rolle zu spielen, durch die Kenntnis des Äußeren einer historischen Person könne man etwas erfahren, das über die Kenntnis ihrer Taten oder Schriften hinausginge. Die-

\footnotetext{
* Der vorliegende Text ist die leicht veränderte Fassung eines Vortrags, den ich im Herbst 1998 anläßlich eines Kolloquiums zum mos maiorum im Rahmen des Sonderforschungsbereichs 537 ,Institutionalität und Geschichtlichkeit" der TU Dresden gehalten habe. Den Einladenden, insbesondere F.-H. Mutschler, und allen Diskussionsteilnehmern gilt mein herzlicher Dank.

Neben den üblichen Abkiirzungen werden die folgenden verwendet:

Zanker = P. Zanker, Grabreliefs römischer Freigelassener, JDAI 90, 1975, 267-315. Kockel $=$ V. Kockel, Porträtreliefs stadtrömischer Grabbauten. Ein Beitrag zur Geschichte und zum Verständnis des spätrepublikanisch-frühkaiserzeitlichen Privatporträts, Mainz 1993.

Verf. = B. Borg, Rez. zu Kockel, GGA 248, 1996, 70-91.
} 
ses Gefühl ist zweifellos bis heute wirksam, denn weshalb sonst sollte man Lexikonartikel oder Klappentexte zu Romanen mit Porträts illustrieren?

Dies bedeutete jedoch, daß sich das Interesse vornehmlich auf solche antiken Bildnisse richtete, die bekannte historische Personen darstellten - oder denen man dies wenigstens unterstellen konnte. Man wird daher leicht einsehen, daß jene Gattung, um die es im folgenden gehen wird, die der Grabreliefs römischer Freigelassener, zunächst wenig interessant eršchien: Einerseits waren sie zum überwiegenden Teil alles andere als ästhetisch ansprechende Kunstwerke; andererseits zeigten sie Personen, welche für die an großen Männern und zentralen historischen Ereignissen orientierte Geschichtsschreibung ausgesprochen ephemere Gestalten waren.

Insofern ist es nicht wirklich verwunderlich, daß die Grabreliefs erstmals in den 70er Jahren unseres Jahrhunderts eine eingehendere Untersuchung erfuhren, als mit der zunehmenden Politisierung der Gegenwart auch das Interesse an der im weiteren Sinne politischen Funktion der antiken, vor allem der römischen Denkmäler stieg und man sich vermehrt auch den Lebensäußerungen weniger prominenter gesellschaftlicher Gruppen zuwandte. 1975 legte erstmals Paul Zanker dar, daß und wie die Grabreliefs als Zeugnisse der Selbstdarstellung einer bestimmten Gesellschaftsschicht, der römischen Libertinen, unsere Kenntnis dieser Schicht, ihrer Werte, Normen und Orientierungspunkte erweitern und ergänzen können. ${ }^{1}$

Diese Grabreliefs, welche meist mehrere Personen im Büstenausschnitt, seltener ganzfigurig wiedergeben, lassen sich seit ca. $80 \mathrm{v}$. Chr. nachweisen. Ihre Blütezeit ist die zweite Hälfte des 1. Jhs. v. Chr., als die Libertinen zunehmend von den sich stabilisierenden Verhältnissen und dem wirtschaftlichen Aufschwung unter Augustus profitierten. ${ }^{2}$ Die Reliefs waren außen an Grabmonumenten angebracht, zumeist an altarähnlichen, kubischen Gräbern, in seltenen, frühen Fällen an hausähnlichen Fassaden (Abb. 1). ${ }^{3}$

Allein die Tatsache ihrer Existenz ist bezeichnend, denn nahmen sie sich im Vergleich mit den Prachtgräbern der Aristokratie auch eher bescheiden

\footnotetext{
${ }^{1}$ Zanker passim. Ähnliche Grabreliefs für Freigelassene wurden auch außerhalb von Rom angefertigt, dazu H. Pflug, Römische Porträtstelen in Oberitalien. Untersuchungen zu Chronologie, Typologie und Ikonographie, Mainz 1989.

${ }^{2}$ Entsprechend sind die Reliefs in dieser Zeit auch nahezu ausnahmslos aus Marmor, während zuvor auch lokale Steinsorten Verwendung fanden. Vgl. Zanker 270f. 281f.; Kockel passim, zu den ältesten Stücken s. 83-93, Gruppe A.

${ }^{3} \mathrm{Zu}$ den zugehörigen Grabtypen s. Zanker 271-281; bes. Kockel 7-14; dazu auch Verf. 82.
} 
aus, so ist der Abstand zu den einfachen Bestattungen in Tonkrilgen oder selbst den Beisetzungen in der Nische eines Columbariums doch bemerkenswert - nicht zu reden von den anonymen Massengräbern der Armen: Man besaB ein eigenes Grabmonument und präsentierte dieses selbstbewuBt zwischen den Gräbern des Adels an prominenter Stelle, an den AusfallstraBen Roms, wo einem die Beachtung der Vorbeiziehenden sicher war. ${ }^{4}$ Die Bildnisse an diesem Monument konnten zudem eine ähnliche Funktion erfüllen wie die öffentlichen Ehrenstatuen der Nobilität; sie hoben den Grabinhaber und seine Familie als Personen mit Würde und Ansehen über ihre ,porträtlosen“ Zeitgenossen hinaus und verglichen sie mit den Ersten der Gesellschaft.

Reliefs und Inschriften der Gräber waren schon von daher kein beliebiger, luxuriöser Schmuck, aber sie vermittelten darüber hinaus auch spezifische Informationen über die Grabinhaber: Zuallererst sollten sie den Stolz auf die Überwindung des Sklavenstatus und der damit verbundenen Restriktionen zum Ausdruck bringen. ${ }^{5} \mathrm{Zu}$ diesen Restriktionen gehörte das Verbot einer rechtmäßigen Ehe und damit das Fehlen einer eigenen, legitimen Familie überhaupt. Bezeichnenderweise stellen daher auch die meisten Reliefs mehrere Personen dar, deren epigraphische wie bildliche Charakterisierung der Konstruktion eines Familienverbandes diente. Dabei konnten auch nicht blutsverwandte Personen, wie etwa der Patron an Stelle eines leiblichen Vaters, einen Platz erhalten. ${ }^{6}$ Besonders wichtig und Krönung aller Hoffnungen war die Geburt eines Sohnes, weshalb die auf den Reliefs dargestellten Kinder auch fast ausschlieBlich Knaben sind. Dieser Sohn wurde mit allen Attributen eines Freigeborenen ausgestattet, der bulla und der toga praetexta (Abb. 2). ${ }^{7}$ In wenigen Fällen war es den Söhnen von Freigelassenen sogar gelungen, zum Militärdienst zugelassen zu werden, welcher weiteren gesellschaftlichen Aufstieg ermöglichte - eine Errungenschaft, die mit entsprechender Deutlichkeit demonstriert wurde (Abb. 3). ${ }^{8}$

\footnotetext{
${ }^{4}$ Vgl. zu den Gräberstraßen auch allgemein: H. v. Hesberg, P. Zanker (Hgg.), Römische Gräberstraßen. Selbstdarstellung - Status - Standard. Kolloquium in München vom 28. bis 30. Oktober 1985, München 1987; H. v. Hesberg, Römische Grabbauten, Darmstadt 1992.

${ }^{5}$ Zum Folgenden s. Zanker 283-306; Kockel 15-55.

${ }^{6}$ Zanker 296-298.

${ }^{7}$ Kockel 53f.; zur bulla s. H. R. Goette, Die Bulla, BJb 186, 1986, 133-164; zur toga s. ders., Studien zu römischen Togadarstellungen, Mainz 1990.

${ }^{8}$ Zanker 303-306; Kockel 24f.
} 
Statusabzeichen der Frauen sind gelegentlich die stola, welche die freigeborene und mit ihrem Mann in manus-Ehe verbundene Gattin auszeichnet, sowie die einen Haarknoten bedeckende vitta als Zeichen der mater familias (Abb. 4). ${ }^{9}$ Statusabzeichen der Männer ist - neben der Familie selbst - die Toga des freien Btirgers. Sie ist gelegentlich in aller Deutlichkeit zu erkennen, doch in den meisten Fällen nur erschließbar: bildlich unterscheidet sie sich oft nicht von einem Pallium oder von der Tracht mancher Frauen. ${ }^{10}$

Spätestens hier zeigt sich die Bedeutung der Inschriften. Zwar tragen heute nur knapp zwei Fünftel der Reliefs Inschriften, diese müssen jedoch in den übrigen Fällen separat am Grab angebracht gewesen sein. Aus ihnen ging der Status der Dargestellten unmiBverständlich hervor: die tria nomina als Zeichen des römischen Bürgers, der Libertinenstatus, gelegentlich der Beruf, sofern es sich um eine angesehene und/oder einträgliche Tätigkeit handelte (ein praeco, eventuell ein lictor, Metallhandwerker, Zimmerleute, ein Getreidehändler, ein faber argentarius, Ärzte und Soldaten), ${ }^{11}$ sowie als wiederum besonders wichtiges Element der Familienstatus. Auf einem Relief in Rom wird der Knabe mit der bulla durch die Inschrift ausdrücklich als f(ilius) bezeichnet, die Frau als uxor: Thre Stellung bliebe ohne die Inschrift unbestimmt, da sie mit ihrem Gatten nicht durch die dextrarum iunctio verbunden ist (Abb. 2). ${ }^{12}$

Der stolz vorgetragene neue gesellschaftliche Status der Libertinen wurde demnach sowohl durch die Inschriften zum Ausdruck gebracht als auch durch die bildlichen Darstellungen. Letztere fügen dem Selbstbild nun allerdings noch weitere Komponenten hinzu, die in den Inschriften nicht erwähnt werden. Wie bereits bemerkt, spielt bei der Darstellung der Männer weniger die Zurschaustellung der Toga als Zeichen des neuen Status eine Rolle als vielmehr die Präsentation der Angemessenheit der Kleidung, die dem Dargestellten den Habitus des ,korrekten Bürgers“ verleiht. ${ }^{13}$ Auch die Soldaten bringen nicht nur ihre formale Position in der gesellschaftlichen

\footnotetext{
${ }^{9}$ Kockel 39-42. 51-53.

${ }^{10}$ Vgl. Zanker 300f,, der zwar die Schwierigkeiten der Unterscheidung erkennt (Anm. 120), aber dennoch auf der spezifischen semantischen Funktion der Toga im Bild beharrt; Kockel 15; dazu Verf. 83-85.

${ }^{11}$ Zanker 279. 298-300; Kockel 54. 245 (Register 4).

${ }^{12}$ Rom, Mus. Vat, Mus. Gregoriano Profano, Inv. 10491: Kockel 141f. Nr. H6 Taf. 51 b. $52 \mathrm{a}-\mathrm{c}$.

${ }^{13}$ Die Bedeutung des, ,korrekten Auftretens" wird auch betont von Zanker 300. 308.
} 
Hierarchie zum Ausdruck, sondern auch ihre militärische virtus. Die Frauenporträts gehen ikonographisch auf hellenistische Statuentypen zurück, die auch in der Oberschicht und noch während der gesamten Kaiserzeit für Porträtstatuen beliebt waren und sich durch bedecktes Haar und die Einbindung der Arme in das Gewand auszeichnen; sinnlichere, verspieltere und luxuriösere hellenistische Typen wurden dagegen nicht kopiert. Zanker hat dies in anderem Zusammenhang sicher zu Recht auf ein Verhaltensideal zurïckgeführt, das man vielleicht mit den Begriffen pudicitia und castitas verbinden kann, das aber in jedem Fall wie die Tracht der Männer ein „,korrektes Auftreten" signalisiert. ${ }^{14}$ Für die Freigelassenen bedeutete diese Tugend nicht nur eine Übernahme von allgemeinverbindlichen Normen der römischen Gesellschaft, sondern auch eine demonstrative Negation der sprichwörtlich unmoralischen Lebensweise der Sklaven. In diesem Zusammenhang wird man auch die dextrarum iunctio nicht allein als Zeichen des matrimonium iustum zu verstehen haben, sondern auch attributiv als symbolischen Ausdruck der concordia und fides der Ehepartner (Abb. 5). ${ }^{15}$ In demselben Sinne ist die Hand oder der Arm auf der Schulter des Partners nicht (allein) Ausdruck ehelicher Zuneigung, ${ }^{16}$ sondern wie auf Münzen und Sarkophagen vor allem Ausdruck der concordia. ${ }^{17}$

Die Freigelassenengrabreliefs und ihre Inschriften bringen somit zweierlei zum Ausdruck: den neu erlangten gesellschaftlichen Status sowie die moralischen Tugenden der Dargestellten. Sie sind damit Teil einer Auseinandersetzung mit den rechtlichen und moralischen Defiziten des Sklavendaseins, welche nun für alle sichtbar überwunden waren: nicht unfrei und rechtlos, sondern frei und mit den Rechten des römischen Bürgers versehen; nicht ehe- und kinderlos, sondern rechtmäßig verheiratet und mit legitimen Kindern gesegnet; nicht freizügig und unmoralisch in der Lebensweise, sondern korrekt und in Eintracht verbunden, sittsam und treu.

Lassen sich alle diese Charakterisierungen demnach in erster Linie als eine re-aktive Negation der Hauptdefizite des Sklavendaseins verstehen, so scheint der semantische Gehalt der Reliefs darüber hinaus zumindest teil-

${ }^{14}$ P. Zanker, Statuenrepräsentation und Mode, in: S. Walker, A. Cameron (Hgg.), Greek Renaissance in the Roman Empire. Papers from the tenth British Museum Classical Colloquium [Bulletin Suppl. 55], London 1989, 102-107.

${ }^{15}$ Kockel 50.

${ }^{16}$ So jedoch Zanker 286f.; Kockel 54.

${ }^{17} \mathrm{Vgl}$. Verf. 85 mit Anm. $46 \mathrm{f}$. 
weise auch eine aktive Wahl weiterer positiver Eigenschaften zu umfassen, die man sich - zumindest im Bild - zulegte. Hierfür sind nun vor allem die Porträts selbst aufschluBreich.

Noch Paul Zanker hatte den Realismus der Darstellungen betont und in ganz traditioneller Weise vermutet: „Die libertini wollten ganz offenbar so gesehen werden, wie sie aussahen."18 Dem widerspricht allerdings die oft starke Typisierung der Bildnisse. ${ }^{19}$ Wie Valentin Kockel in seiner monographischen Behandlung der Reliefs deutlich gemacht hat, verwenden die Darstellungen tatsächlich ein relativ überschaubares Repertoire physiognomischer Grundtypen, die nur einzeln betrachtet den Eindruck großer Individualität vermitteln. ${ }^{20}$ Interessant ist an dieser Beobachtung vor allem zweierlei. Zum einen finden sich dieselben physiognomischen Grundtypen werkstattïbergreifend, so daß sie nicht allein mit handwerklichen Traditionen erklärt werden können; zum andern finden sich dieselben Typen auch unter den Porträts der Nobilität und der berühmtesten Männer des Staates. Dieses Phänomen läßt sich vielleicht auf der Grundlage einiger allgemeinerer Überlegungen zu den römischen Porträts besser verstehen.

Das eingangs erwähnte diffuse Gefühl, die Kenntnis der äußeren Erscheinung einer Person könne uns etwas über deren Wesen und Charakter erzählen, hat bereits in der Antike einen expliziten Ausdruck in physiognomischen Schriften gefunden. ${ }^{21}$ Bei der modernen Beschäftigung mit Porträts hat man zwar auf diese offensichtlich absurden und in sich oft widersprüchlichen Theorien nicht zurückgegriffen, doch sind die spontanen, auf einem allgemeinen Eindruck beruhenden Deutungen, wie sie in diesem Jahrhun-

${ }^{18}$ Zanker 312; ebenso ders., Zur Rezeption des hellenistischen Individualporträts in Rom und in den italischen Städten, in: ders. (Hg.), Hellenismus in Mittelitalien. Kolloquium in Göttingen vom 5. bis 9. Juni 1974, Göttingen 1976, 592-596, wo eine ähnliche Deutung auch den vergleichbaren Porträts der Nobilität unterstellt wird (ebd. 591f.). Das Mißverständnis beruht m.E. darauf, daß Zanker nur den aus dem hellenistischen Porträt abgeleiteten Pathosformeln eine spezifische semantische Funktion zubilligt, nicht aber den Formeln der naturalistischen Porträtrichtung; s. dazu das Folgende.

${ }^{19}$ Mit den im folgenden entwickelten Argumenten soll keineswegs bestritten werden, daB antike Porträts - und insbesondere die Freigelassenenporträts auf den Grabreliefs - den Dargestellten nicht auch ähnlich gewesen sein konnten; es kommt hier lediglich darauf an, zu zeigen, daß Ähnlichkeit jedenfalls nicht die einzige und oft vielleicht nicht einmal die vorrangige Darstellungsabsicht gewesen ist.

${ }^{20}$ Kockel 62-67.

${ }^{21}$ E. C. Evans, Physiognomics in the Ancient World, TAPhS 59, 1969; zu Polemons Physiognomik und ihren Quellen s. M. W. Gleason, Making Men. Sophists and selfpresentation in ancient Rome, Princeton (New Jersey) 1994, bes. 29-54. 
dert in Ludwig Curtius ihren prominentesten Vertreter gefunden haben, ebenfalls problematisch. ${ }^{22}$ Ich will nicht noch einmal das mittlerweile etwas strapazierte Beispiel seiner berïhmten Deutung des Pompeius-Porträts wiederholen, sondern zum Beweis der Tatsache, daß die seinem Vorgehen zugrundeliegenden Voraussetzungen auch in den 60er Jahren noch geläufig waren, ein jüngeres Beispiel zitieren: So meinte eine renommierte deutsche Porträtforscherin über ein Bildnis des Nero im Thermenmuseum: „[Das Bildnis] enthüllt in jedem Zug seinen pathologischen Charakter. Als letzter Abkömmling der julisch-claudischen Herrscherfamilie, allerdings nur mütterlicherseits, trägt er das Erbe einer langen Reihe von Familienheiraten in sich. Seine Züge sind aufgeschwemmt und verwischt, klein und aufgeworfen ist der Mund, die kurzsichtigen Augen (...) eingesunken. Eine unmenschliche, grausame Freundlichkeit und Unberechenbarkeit spiegeln sich in ihnen. Die vollen, dichten Haare, die in schöngeschwungene, lange, unregelmäBig gelegte Locken frisiert sind, dienen dazu, das Eitle seines Charakters zu unterstreichen (...).6.23 Den Vorwurf eines plumpen Psychologismus hätte sie sicherlich weit von sich gewiesen, denn immerhin stimmt ihre Darstellung mit den ebenfalls wenig schmeichelhaften Charakterisierungen dieses Kaisers durch die antiken Schriftsteller überein und scheint somit eine verläßliche Grundlage zu besitzen. Um so weniger schien man die impliziten Voraussetzungen solcher Deutungen in Frage stellen zu müssen: zum einen die Annahme, aus Physiognomie und Mimik einer Person ließe sich auf gewisse Charakterzïge schließen, zum anderen die eingangs erwähnte Vermutung, die Porträts gäben die betreffenden Personen wieder, wie sie tatsächlich ausgesehen haben.

Doch hatte ebenfalls in den 60er Jahren das Fundament dieser Vorgehensweise bereits zu wackeln begonnen, als erstmals der systematische Versuch unternommen wurde, die römischen Porträts zu benennen und zu datieren. Dabei stellte sich rasch heraus, daß sich die plastischen und die Münzbildnisse der Herrscher und Herrscherinnen zu Gruppen zusammenstellen ließen, die so weitgehend übereinstimmten, daß sie auf ein gemeinsames Urbild zurückgehen mußten. Gleichzeitig ermöglichte diese Feststellung eine zuverlässigere Unterscheidung zwischen Kaiser- und Privatporträts und öffnete den Blick für ein interessantes Phänomen: Privatpersonen imitierten

${ }^{22} \mathrm{Vgl}$. zu den modemen physiognomischen Deutungen L. Giuliani, Bildnis und Botschaft, Frankfurt a. M. 1986, 11-51.

${ }^{23}$ H. v. Heintze, Römische Porträt-Plastik aus sieben Jahrhunderten, Stuttgart 1961, 9 f. 
nicht nur die Frisurmode des Kaiserhauses, sondern glichen sich gelegentlich auch physiognomisch den Mitgliedern der Herrscherfamilie an. Die Bildnisse sowohl der Herrscher als auch der Privatpersonen konnten daher nicht rein mimetische Abschilderungen tatsächlicher Physiognomien sein, sondern wollten offenbar auch bestimmte Aussagen vermitteln. Daß diese Aussagen - zumindest nach der Beurteilung der Zeitgenossen - aber immer positive Aussagen sein mußten, ergab sich schon aus der öffentlichen Aufstellung der Bildnisse.

Damit war natürlich auch die gängige Deutung des Neroporträts hinfällig. Spätestens jetzt machte man sich klar, daß die Charakterisierung Suetons zwar bei manchen Zeitgenossen auf eine gewisse Zustimmung gestoBen sein dürfte, aber keineswegs die einzige Ansicht über den Kaiser repräsentierte - und schon gar nicht die Ansicht des Kaisers selbst. Für die Porträts des Herrschers bedeutete dies aber, daB die Aussageabsicht, wie immer man sie präzisieren mochte, ${ }^{24}$ eine positive sein mußte. Und mehr noch: daB diese Aussage auch als positiv verstanden wurde, denn wie sonst sollte man sich erklären, daß Privatpersonen sich in ihren Porträts der Erscheinung des Kaisers anglichen ? $^{25}$

Mit der bloßen Feststellung des Vorhandenseins einer programmatischen Absicht von Porträts war es aber natürlich nicht getan; man wollte auch zum Inhalt der Aussagen vordringen, ein Unterfangen, bei dem oft die bisherige "Methode" des unmittelbaren Zugangs beibehalten und nun lediglich negative Deutungen vermieden wurden. Tatsächlich wird dabei jedoch ein grundsätzlicheres Problem übersehen: Zwar scheinen mimische Züge oft nur ein begrenztes Deutungsspektrum zuzulassen; zu denken wäre etwa an das Lachen, das wir schwerlich als Ausdruck von Ärger auffassen, oder an Stirnrunzeln, das wir wohl kaum mit freudiger Zustimmung assoziieren werden. Doch zeigt ein Blick auf andere Kulturkreise, wie sehr wir unter Umständen mit ad hoc-Deutungen in die Irre gehen können, wie kulturabhängig die Interpretation scheinbar universaler mimischer Bewegungen tatsächlich ist. Nelson Goodman zitiert beispielsweise aus einem Vortrag des Anthropologen Ray L. Birdwhistell: „Es gibt ebensowenig Körperbewe-

\footnotetext{
${ }^{24} \mathrm{Zu}$ den Neroporträts und ihrer Deutung vgl. zusammenfassend $\mathrm{M}$. Bergmann, Die Strahlen der Herrscher. Theomorphes Herrscherbild und politische Symbolik im Hellenismus und in der römischen Kaiserzeit, Mainz 1998, 147-149; zum Frisurentypus allgemein P. Cain, Männerbildnisse neronisch-flavischer Zeit, München 1993, 58-68. 88-95.

${ }^{25}$ Zum Privatporträt neronischer Zeit s. P. Cain (wie Anm. 24), a.O.
} 
gungen, Gesichtsausdrücke oder Gesten, die auf der ganzen Welt identische Reaktionen hervorrufen, wie es universale Wörter, Lautkomplexe gibt, die auf der ganzen Welt dieselbe Bedeutung tragen. Ein Körper kann vor Kummer gebeugt sein, in Demut, vor Lachen oder zum Angriff bereit. Ein Lächeln bedeutet in einer Gesellschaft Freundlichkeit, in einer anderen Verlegenheit, und in wieder einer anderen kann es eine Warnung enthalten, daß Feindseligkeit und Angriff folgen werden, wenn sich die Spannung nicht auflöst. ${ }^{626}$ Des weiteren erinnert Goodman daran, wie schwierig es für das westliche Publikum der ersten qualitätvollen japanischen Filme war herauszufinden, welche Emotionen die Schauspieler zum Ausdruck bringen wollten, ob ein bestimmter Gesichtsausdruck beispielsweise „Qual oder $\mathrm{HaB}$ oder Angst oder Entschlossenheit oder Verzweiflung oder Verlangen ausdrückte“. ${ }^{27}$ Selbst wenn wir eine bestimmte Mimik erkannt, beschrieben und benannt haben, ergibt sich erst aus dem kulturellen Kontext die eigentliche Bedeutung dieser „Geste“.

$\mathrm{DaB}$ nun aber die römische Gesellschaft eine uns in vieler Hinsicht fremde ist, belegen nicht zuletzt die von uns heute belächelten, ehemals aber nicht nur ernst gemeinten, sondern geradezu für evident gehaltenen physiognomischen Deutungen durch Curtius, v. Heintze und andere. Das vielzitierte Wort von Uvo Hölscher über das „nächste Fremde“ bringt die gefährliche Ambivalenz in unserem Verhältnis zur klassischen Antike zum Ausdruck: Aufgrund unserer Tradition empfinden wir eine gewisse Nähe zur antiken Kultur, die uns andererseits die Fremdheit derselben allzu leicht vergessen läßt. Wie wenig unmittelbar unser Zugriff auf antike Porträts als historische Dokumente tatsächlich ist, mag ein Blick auf das Porträt Octavians verdeutlichen (Abb. 6): Sein gesträubtes Stirnhaar beispielsweise hat für einen unvoreingenommenen Betrachter vielleicht noch die Anmutungsqualität „dynamisch“ (mancher Museumsbesucher hält Octavian jedoch schlicht für unfrisiert); daß es sich aber tatsächlich um ein Alexander-Zitat handelt, erkennt nur derjenige, der einerseits mit dessen Ikonographie und andererseits mit der Art und Weise der Selbststilisierung und dem Umgang

${ }^{26}$ N. Goodman, Languages of Art. An Approach to a Theory of Symbols, Indianapolis 1968; deutsch: Sprachen der Kunst. Entwurf einer Symboltheorie, Frankfurt a. M. 1997, $56 \mathrm{f}$.

${ }^{27}$ Goodman (wie Anm. 26), 56. 
mit Bildzitaten im antiken Porträt überhaupt vertraut ist. ${ }^{28}$ Vergleichbare Mißverständnisse sind archäologische Realität. Die mit den Rufnamen Cato und Porcia versehenen Porträts eines Grabmals im Vatikan (Abb. 5) gelangten als Urbild eines römischen Ehepaares und römischer Tugenden bis in die Schulbücher. Tatsächlich handelt es sich jedoch der zugehörigen Inschrift zufolge um einen M. Gratidius Libanus und seine liberta Chrite, deren Abstammung alles andere als italisch-römisch gewesen sein dürfte. ${ }^{29}$

Diesen Überlegungen trug Luca Giuliani Rechnung, indem er in seinem Buch mit dem aufschluBreichen Titel „Bildnis und Botschaft" die programmatische Darstellungsabsicht republikanischer Porträts aufgezeigt und einen neuen Interpretationsansatz vorgeschlagen hat. ${ }^{30}$ Bei genauerer Betrachtung lassen nämlich die scheinbar schonungslos realistischen und individuellen Portrăts der spätrepublikanischen Oberschicht bestimmte immer wiederkehrende mimische Formeln erkennen, die Giuliani „pathognomisch“ nannte und die den individuellen Zügen der einzelnen Personen attributiv hinzugefügt sind. Diese mimischen Formeln faßte Giuliani als eine Art Gesten auf, welche ähnlich den Gesten von Rednern in öffentlichen Versammlungen weitgehend kanonisiert und in ihrer Bedeutung und ihrem Platz im Wertekanon angemessenen Benehmens festgelegt waren. Von den zahlreichen antiken Quellen, die ein solches Vorgehen rechtfertigen, sei hier nur aus Ciceros In Pisonem zitiert. Dort wird der angesehene Konsul L. Calpurnius Piso Caesonianus beschuldigt: „Siehst du jetzt, du Ungeheuer, merkst du jetzt, was die Leute gegen deine finstere Miene haben? (...) Die Augen, die Stirn, überhaupt die ganze Miene, die ja ohne Worte die Gesinnung ausspricht - das hat die Leute zum Irrtum verleitet, das hat alle, die dich nicht kannten, getäuscht, betrogen, hinters Licht geführt. Nur wenige von uns kannten deine schmutzigen Begierden, deinen schwerfälligen Geist, deine plumpe, stammelnde Ausdrucksweise. Nie hatte man auf dem Forum deine Stimme vernommen, nie deinen Rat erprobt; man wuBte von keiner beachtlichen oder gar glänzenden Tat, sei es in der Heimat, sei es im Felde.

${ }^{28} \mathrm{Zu}$ Octavians Porträt s. K. Fittschen, P. Zanker, Katalog der römischen Porträts in den Capitolinischen Museen und den anderen kornmunalen Sammlungen der Stadt Rom. Bd. I: Kaiser- und Prinzenbildnisse, Mainz ${ }^{2} 1994$, 1f. (Zanker); D. Boschung, Die Bildnisse des Augustus. Das römische Herrscherbild 1,2, Berlin 1993 (mit problematischer Typeneinteilung); grundlegend P. Zanker, Studien zu den Augustus-Porträts I, Der ActiumTypus, Göttingen 1973.

${ }^{29}$ Zanker 285f.; Kockel 188-190 zu L 19 Taf. 105.

${ }^{30}$ Giuliani (wie Anm. 22), passim. 
Du hast dir die Ämter erschlichen, weil die Leute sich täuschen ließen." (Übers. M. Fuhrmann). ${ }^{31}$ Schon in jungen Jahren habe Piso durch seine „mit Berechnung geheuchelte strenge Miene“ die Leute getäuscht. ${ }^{32}$ Den von Giuliani nachgewiesenen bildlichen Chiffren bestimmter mimischer Züge scheinen demnach sogar teilweise tatsächliche Verhaltensmaßregeln entsprochen zu haben.

Gegenüber älteren Kommentaren ist entscheidend, daß Giuliani das bekannte Wertesystem weder pauschal auf eine ganze gesellschaftliche Gruppe bezieht noch auf einen allgemeinen Eindruck, den die Bildnisse unserem voreingenommenen Blick vermitteln, sondern daB er einzelne Werte und Tugenden mit konkreten Bildformeln verbindet und damit eine Art Leseanleitung für die Porträts der republikanischen Oberschicht erstellt. Während die Zeitgenossen die Signale zweifellos aus ihren Sehgewohnheiten und ihrer Vertrautheit mit den allgemeinen Verhaltensnormen heraus intuitiv erfaßten und nicht bewußt entziffern mußten, eröffnet der von Giuliani vorgeschlagene Weg eine differenziertere Annäherung an die verschiedenen Weisen der Selbstdarstellung auch für uns Heutige. ${ }^{33}$

Auf der Grundlage dieser Thesen lohnt nun noch einmal ein Blick auf die Grabreliefs der Libertinen, denn wie bereits angedeutet, besitzen viele der

${ }^{31}$ Cic. Pis. 1: iamne vides, belua, iamne sentis quae sit hominum querela frontis tuae? (...) oculi, supercilia, frons, voltus denique totus, qui sermo quidam tacitus mentis est, hic in fraudem homines impulit, hic eos quibus eras ignotus decepit, fefellit, induxit. pauci ista tua lutulenta vitia noramus, pauci tarditatem ingeni, stuporem debilitatemque linguae. numquam erat audita vox in foro, numquam periculum factum consili, nullum non modo inlustre sed ne notum quidem factum aut militiae aut domi. obrepsisti ad honores errore hominum (...); dazu Giuliani (wie Anm. 22), $227 \mathrm{f}$.

${ }^{32}$ Cic. p. red. in sen. 13: nam ille alter Caesoninus Calventius ab adolescentia versatus est in foro, cum eum praeter simulatam versutamque tristitiam nulla res commendaret, non consilium, non dicendi facultas, non rei militaris, non cognoscendorum hominum studium, non liberalitas; Giuliani (wie Anm. 22), 228; weitere Beispiele ebd. passim.

${ }^{33}$ Dieser grundsätzliche Wert der Methode wird durch die m.E. berechtigte Kritik an manchen Einzelinterpretationen Giulianis ebenso wenig geschmälert wie durch die Feststellung, man habe viele der nun mit bestimmten mimischen Formeln verbundenen Eigenschaften schon immer erkannt (s. bes. K. Fittschen, Pathossteigerung und Pathosdämpfung, AA 1991, 253-270): Die berechtigte Kritik an der Behandlung einzelner Bildnisse bezieht sich in erster Linie auf deren Datierung und namentliche Identifizierung, die mit den Deutungen der Bildforme(1)n gelegentlich in Zirkelschlüsse geraten. DaB an bestimmten Deutungen nie ein Zweifel bestanden hat, belegt nicht die Überfluissigkeit eines methodischeren Vorgehens, sondern nur die Tatsache, daß manche (aber eben keinesfalls alle!) intuitiven Deutungen richtig gewesen sind, wobei sich ihre Richtigkeit freilich erst auf dieser methodischen Grundlage wirklich erweist. 
physiognomischen Typen dieser Reliefs eine mehr oder weniger deutlich ausgeprägte Ähnlichkeit mit Porträts von bedeutenden Persönlichkeiten der späten Republik. ${ }^{34}$ Im Einzelfall mag es durchaus sein, daB beispielsweise ein physiognomischer Typus, der dem Caesarporträt Typus Tusculum aus den 40er Jahren ähnlich ist und bereits zu den wüstesten Caesaridentifizierungen seitens der modernen Forschung verführt hat, mit einer persönlichen Verehrung Caesars durch diejenigen Zeitgenossen zusammenhängt, welche ihr Bild dem seinen anglichen; und dasselbe mag auch für die Angleichung mancher Freigelassener an den später erfundenen Bildnistypus des Caesar gelten (Abb. 7 und 8). ${ }^{35}$ Ebensogut ist es aber möglich, daB solche Angleichungen nicht der Person selbst galten, sondern den in ihrer äußeren Erscheinung ausgedrückten Werten und Idealen. Dafür spricht, daß die Ähnlichkeiten in den meisten Fällen relativ allgemeiner Natur sind, aber auch, daß man offenbar solche physiognomischen Typen und Vorbilder bevorzugte, die den bürgerlichen, „staatstragenden“ Tugenden der Aristokratie Ausdruck verliehen. Wer sich in der Art des Cicero darstellen lieB, ${ }^{36}$ wollte damit vielleicht nicht unbedingt seine direkte Anhängerschaft und Bewunderung zum Ausdruck bringen, sich aber als Mensch des Geistes zu erkennen geben oder andere durch Cicero exemplarisch verkörperte Eigenschaften auf sich übertragen, welche diese auch immer im einzelnen gewesen sein mögen.

Die schonungslose Präsentation des Alters (Abb. 3), die so oft allein als Zeichen einer ,realistischen“ Darstellungsweise (miB)verstanden wurde, ergibt einen neuen Sinn, wenn man sie mit den Schlagwörtern der zeitgenössischen Rhetorik auf die mit dem Alter besonders verbundenen Tugenden consilium, ratio, sapientia, sententia oder auctoritas verbindet, vielleicht aber auch im Sinne einer Art Leistungsporträt auf ihre während eines langen Lebens erworbenen Verdienste bezieht: In keiner Porträtgattung werden die Alterszeichen so schonungslos dargestellt wie auf den Freigelassenenreliefs. $^{37}$

Während energetischere Bildnisse, die noch entfernt an Porträts wie die eines unbekannten, wegen der Replikenzahl aber bedeutenden Mannes des

\footnotetext{
34 Andeutungsweise bereits bei Giuliani (wie Anm. 22), $232 \mathrm{f}$.

${ }^{35}$ Beispiele bei Kockel 64f., der das Phänomen jedoch nicht deutet; Lit. zu den Caesarporträts ebd. Anm. 540. 543.

${ }^{36} \mathrm{Vgl}$. Kockel 65 .

${ }^{37}$ Giuliani (wie Anm. 22), 225-233.
} 
späten 2. Jhs. erinnern und mit der Weisheit des Alters zugleich fortitudo, Dynamik und Tatkraft verbinden mögen, ${ }^{38}$ selten und zumeist früh sind (Abb. 9) ${ }^{39}$ führt uns die Mehrzahl der Männer eine beruhigte, ernsthafte Miene vor, wie sie uns bei Cicero, Caesar (Abb. 7), Crassus (Abb. 10) oder Agrippa begegnet. ${ }^{40}$ So erinnern etwa die kontrahierten Brauen, die gepreßten Lippen, die gesamte Mimik des Crassus-Porträts an das obige Zitat aus der Cicerorede und können mit den Schlagwörtern gravitas, severitas und constantia verbunden werden, die auch die dignitas eines senatorischen Amtes gewährleisteten (Abb. 10). ${ }^{41}$ Wenngleich dieses Amt außerhalb der Reichweite der auf unseren Reliefs Porträtierten gelegen hat, ehren doch die hierfür qualifizierenden Eigenschaften auch einen normalen Bürger und pater familias (Abb. 11). Welche Gründe auch immer die in der gesamten mittelmeerischen Kunst zu beobachtende Pathosdämpfung seit dem zweiten Drittel des 1. Jhs. haben mag, in Rom muß der ruhige und gefaßte Ausdruck des Caesarporträts (Abb. 7) und verwandter Bildnisse, der auf jede herrscherliche Allüre verzichtete, als Zeichen von temperantia und mansuetudo, modestia und constantia aufgefaßt worden sein, die gegen die Greuel des Bürgerkrieges immer wieder beschworen wurden. ${ }^{42}$ Gerade sie ziemten sich aber nicht nur für Imperatoren, sondern für jeden guten Staatsbürger und waren somit auch für unsere Libertinen imitationswürdig.

Auffällig ist, daß echte Pathosformeln hellenistischer Tradition fast gänzlich fehlen: Nirgends finden wir die pathetische Kopfwendung des Pompeius oder des jungen Octavian (Abb. 6). Gesteigerte Selbstinszenierung und am Habitus hellenistischer Herrscher orientierte Demonstration der virtus imperatoria entsprachen nicht der Rolle eines Libertinen. Das gesträubte Stirnhaar eines jungen Mannes auf einem Relief in der Villa Wolkonsky (Abb. 12), das durch das Pompeiusporträt (Abb. 13) angeregt

${ }^{38}$ Die Bildnisse tragen den Rufnamen des Postumius Albinus; vgl. Giuliani (wie Anm. 22), 190-199 mit der nicht zu sichernden Deutung auf M. Porcius Cato d. Ä.

${ }^{39} \mathrm{Vgl}$. bes. ein ganzfiguriges Relief in Rom, Mus. Cap., Pal. Cons., Pr.N II,24, Inv. 2142: Kockel 94f. Nr. Bl Taf. 10a. 12a-b. 14a-b (hier Abb. 9). Daß auch dieses Bildnis verglichen mit dem sog. Postumius Albinus deutlich beruhigt ist - was auch den Vergleich mit dem auch von Kockel herangezogenen sog. Feldherrn Tivoli überzeugend erscheinen läßt -, soll hier nicht bestritten werden, doch scheint es zugleich im Vergleich mit den übrigen Bildnissen der Gruppe noch verhältnismäßig bewegt zu sein.

${ }^{40}$ Kockel 64-66.

${ }^{41}$ Giuliani (wie Anm. 22), 223-225. 233-238; zu den Grabreliefs Kockel 64.

${ }^{42}$ Giuliani (wie Anm. 22), 200-220. 
sein mag, ${ }^{43}$ ist eine Ausnahme und erscheint vor dem Hintergrund der übrigen Bildnisse beinahe ambitiös. Es ist bezeichnend, daB die wenigen Soldaten auf den Reliefs (Abb. 3) keine der gesteigerten Pathosformeln der militärischen Führer übernehmen. Die „Botschaften“ des hellenistischen Herrscherporträts und seiner republikanischen Nachfolger dürften ebenso wie zunächst die Ideołogie der augusteischen Idealporträts den Ehrgeiz der meisten Libertinen doch überstiegen haben.

Es ist in diesem Rahmen weder möglich noch nötig, die Berechtigung jeder einzelnen der konkreten Lesarten mimischer Formeln durch Giuliani zu prüfen, diese auf einzelne Köpfe der Freigelassenenreliefs zu übertragen (wobei die Methode ohnehin nicht überstrapaziert werden sollte) oder den ideologischen Rahmen, in dem erst die genannten Schlagwörter ihre wahre Bedeutung entfalten, detailliert darzulegen. Wichtig scheint mir jedoch folgendes zu sein: Die zunächst rein abstrakten Wertbegriffe und Normen der römischen Aristokratie lassen sich in Beziehung zu konkreten Verhaltensidealen und Habitus, also zur kulturellen Praxis bringen, welche ja erst über ihre Wirksamkeit oder Unwirksamkeit im gesellschaftlichen Leben entscheidet. Diese äußerlichen Ausdrucksweisen in Verhalten und Habitus spiegeln sich wiederum in der Bildnisrepräsentation wider, vor allem in jener der Nobilität und der politischen und gesellschaftlichen Protagonisten. Wenn die Libertinen sich daher an diese Bildnisse anglichen, so belegt dies aus ihrer eigenen Perspektive die Akzeptanz der vom Adel propagierten Normen zumindest in ihren Grundzïgen. Zugleich erweist sich aus der Perspektive der Nobilität die Vermittlung ihrer Normen auch an andere gesellschaftliche Schichten als außerordentlich erfolgreich, sowohl was die Inhalte, als auch was die Vermittlungsstrategien angeht.

${ }^{43}$ Kockel 33 mit Anm. 261; 100 za B 8 Taf. 15c. 16a-d; zum Pompeiusporträt s. bes. M. Bentz, Zum Porträt des Pompeius, RM 99, 1992, 229-246. 


\section{ABBILDUNGSVERZEICHNIS}

Abb. 1: Grabbauten an der Via Statilia, Rom; nach: Kockel Taf. 1,1

Abb. 2: Grabrelief der Servilii; Rom, Vat., Mus.Greg.Prof. Inv. 10491; nach: F. Sinn, Vatikanische Museen. Museo Gregoriano Profano ex Lateranense. Die Grabdenkmäler 1, Reliefs Altäre Umen, Mainz 1991, Abb. 14

Abb. 3: Grabrelief der Gessii; Boston, MFA Inv. 37.100; nach: Kockel Taf. 68a

Abb. 4: Grabrelief; Rom, Pal. Cons., Mus. Nuovo VI,12, Inv. 2231; nach: Kockel Taf. 31a

Abb. 5: Grabmal der Gratidii; Rom, Vat., Sala dei Busti 388; nach: Kockel Taf. 105a

Abb. 6: Porträt des Octavian, La Alcudia, Privatbes.; nach: Inst.Neg. DAI Madrid

Abb. 7: Porträt des Caesar im Typus Pisa/Chiaramonti: Rom, Vat., Sala dei Busti, Inv. 713; nach: F. S. Johansen, The Portraits in Marble of Gaius Julius Caesar: A Review, in: Ancient Portraits in the J. Paul Getty Museum, Vol. 1, Malibu 1987, 18 Abb. 1

Abb. 8: Reliefportrait des P. Furius (Det.): Rom, Vat., Mus.Greg.Prof. Inv. 10464; nach: Sinn a.O. Abb. 21

Abb. 9: Ganzfiguriges Grabrelief eines Ehepaars, Det.; Rom, Pal. Cons., BrN, Inv. 2124; nach: Kockel a.O. Taf. 12a

Abb. 10: Porträt des Crassus: Gipsabguß München nach Paris, Louvre Inv. Ma 1220; nach: D. Boschung, Überlegungen zum Liciniergrab, JDAI 101, 1986, 279 Abb. 27

Abb. 11: Grabrelief der Licinii, Alatri, Corso Vittorio Emanuele 41; nach: Kockel a.O. Taf. 41d

Abb. 12: Grabrelief, Det.; Rom, Villa Wolkonsky; nach: Kockel Taf. 16b

Abb. 13: Porträt des Pompeius; Venedig, Mus. Arch. Inv. 62; nach: M. Bentz, Zum Porträt des Pompeius, RM 99, 1992, Taf. 68a 


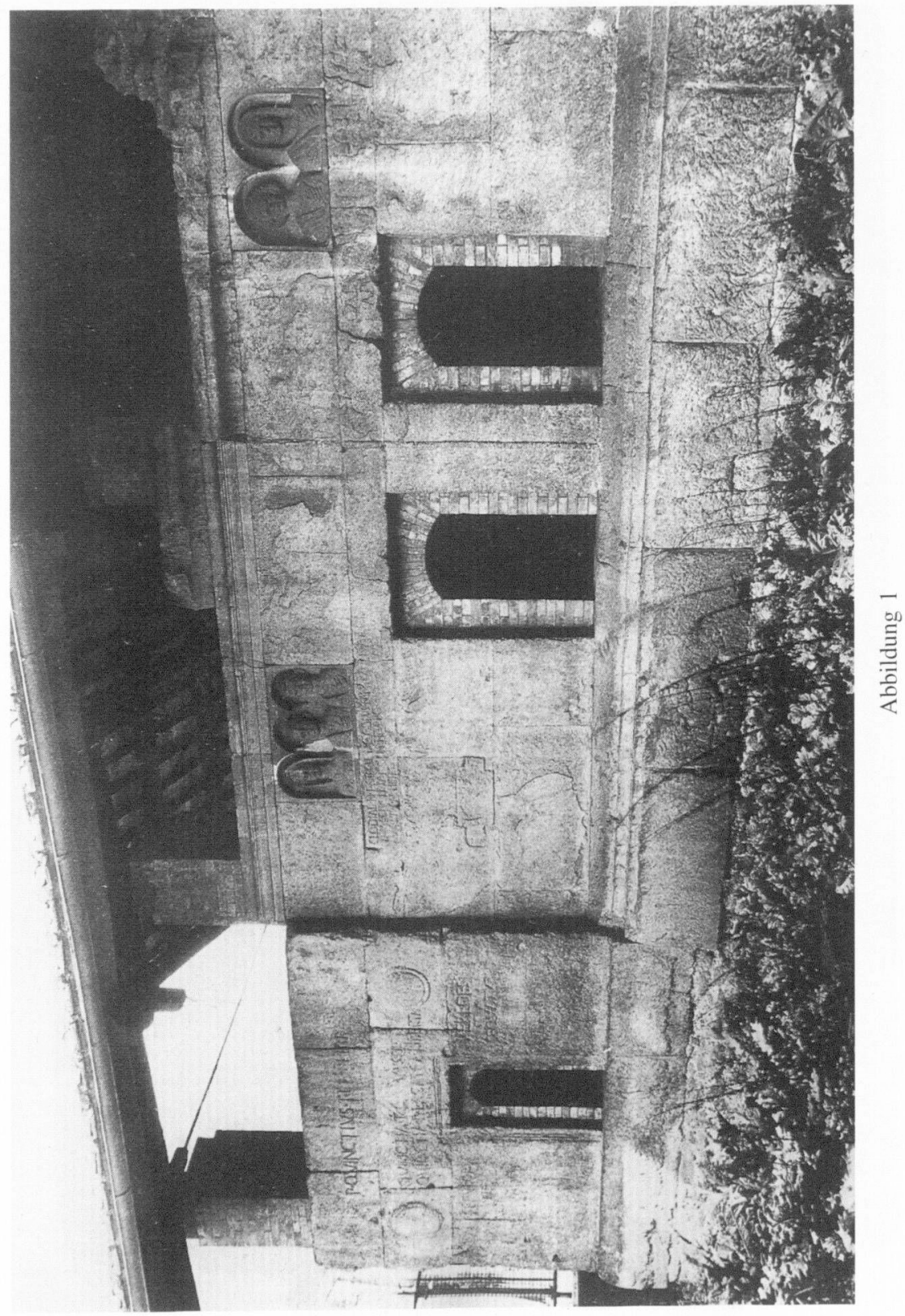




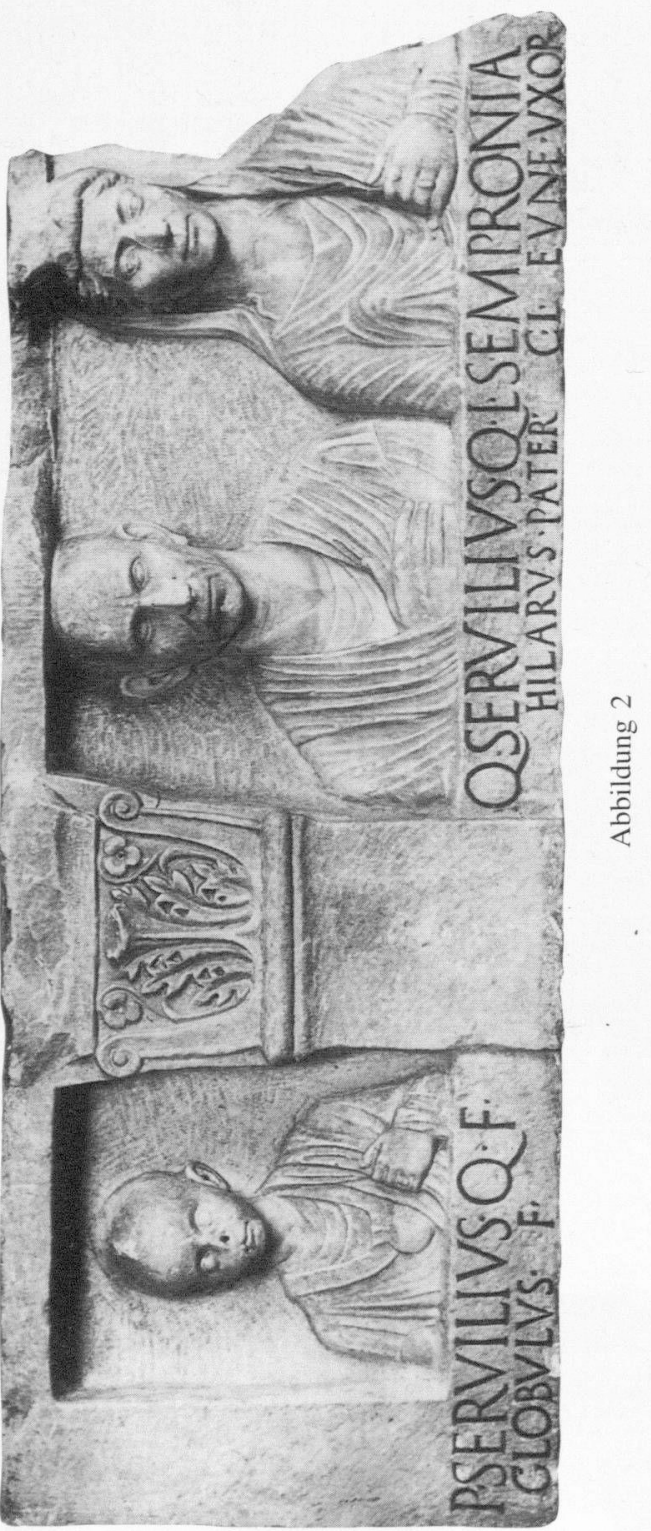



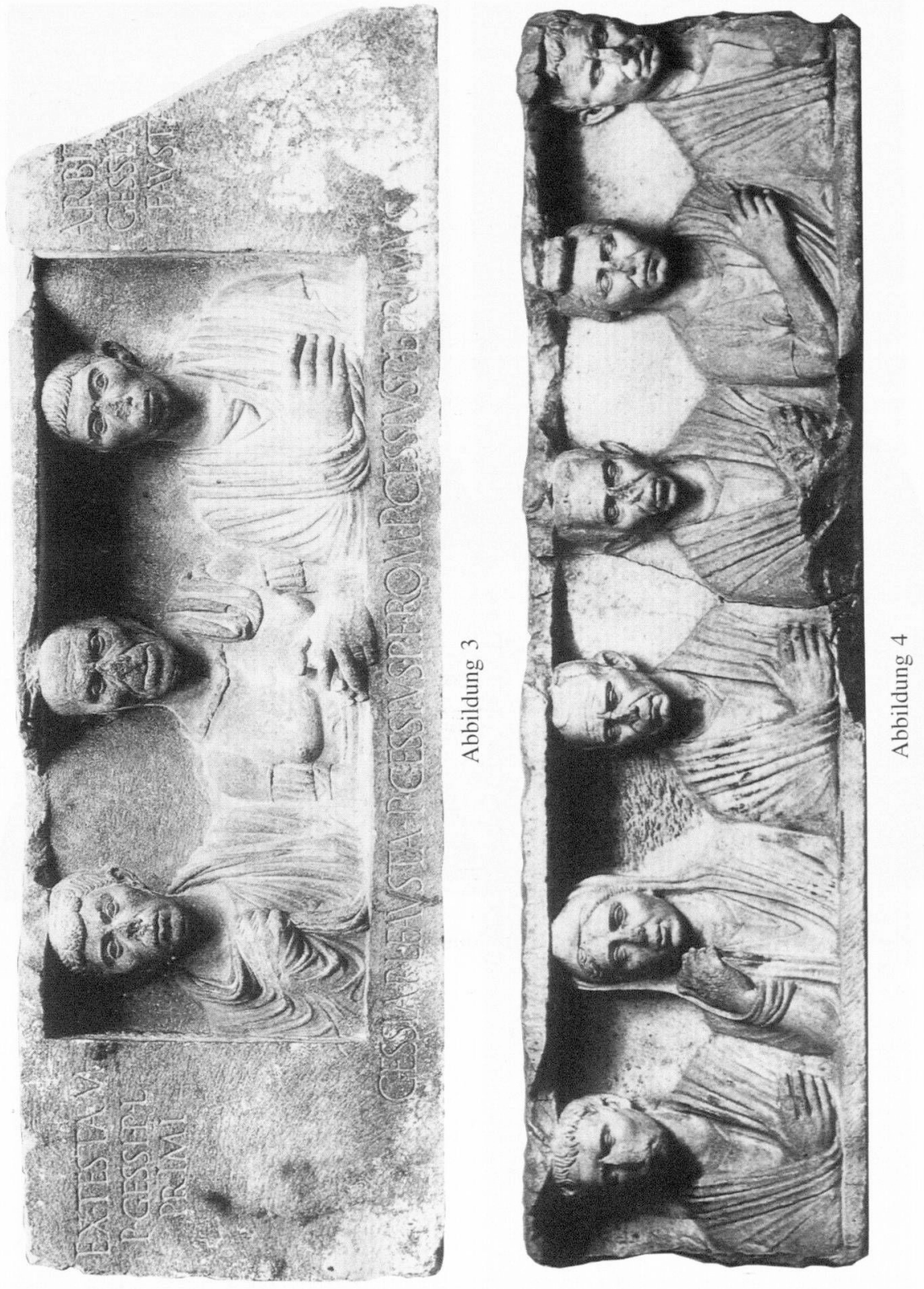


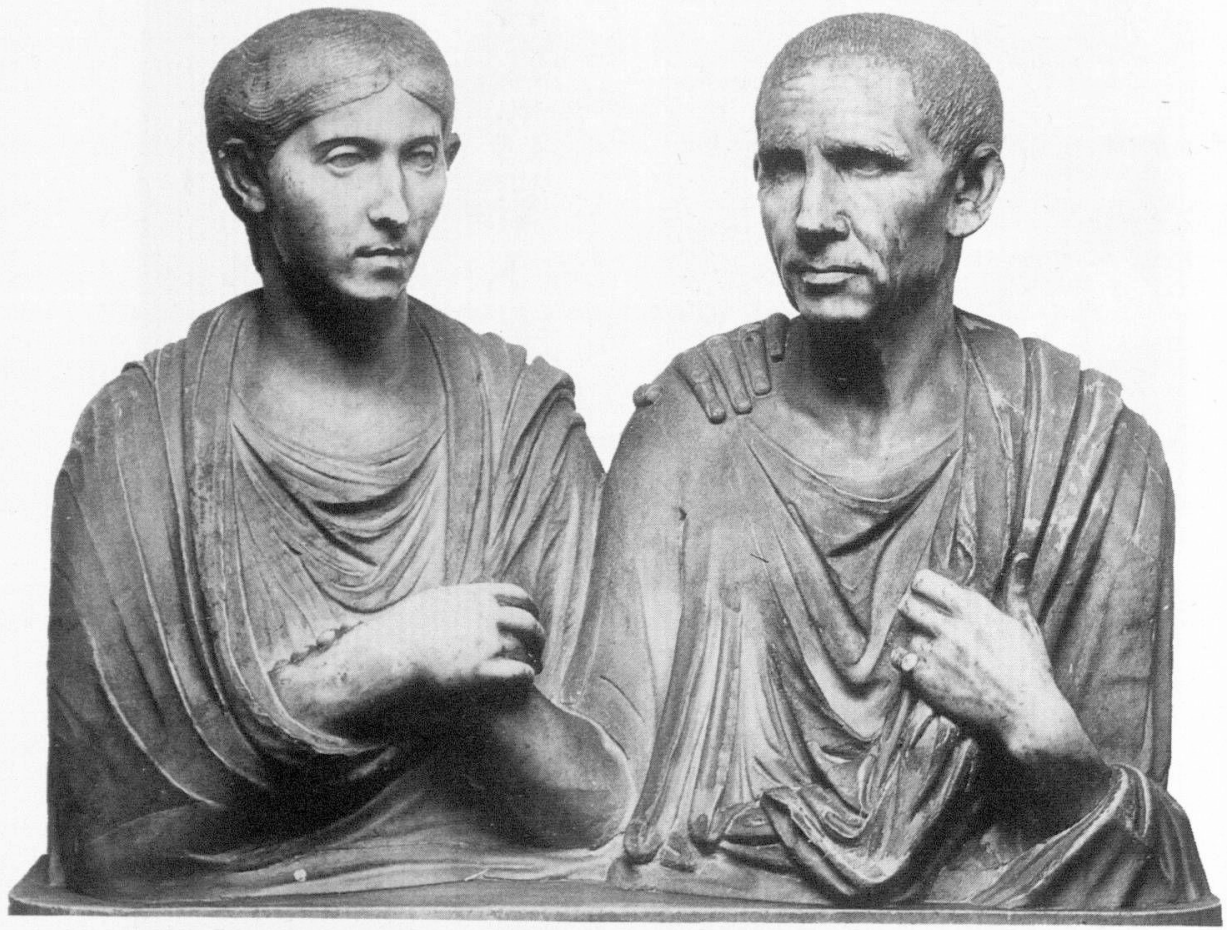

Abbildung 5 


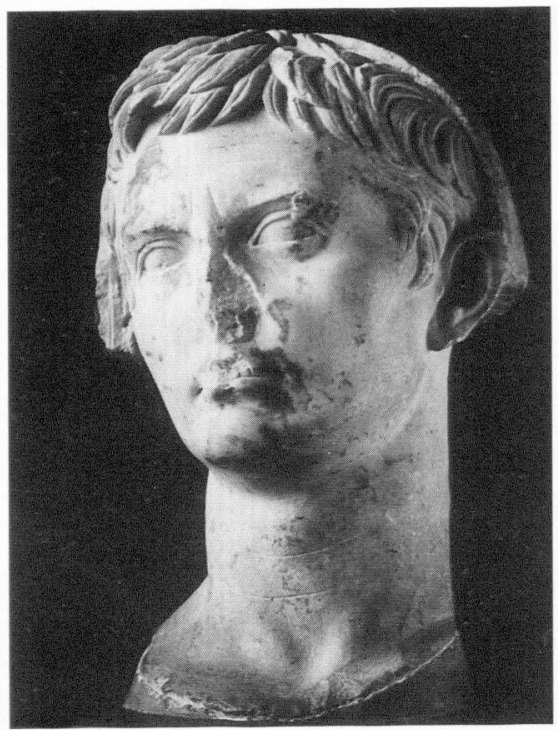

Abbildung 6

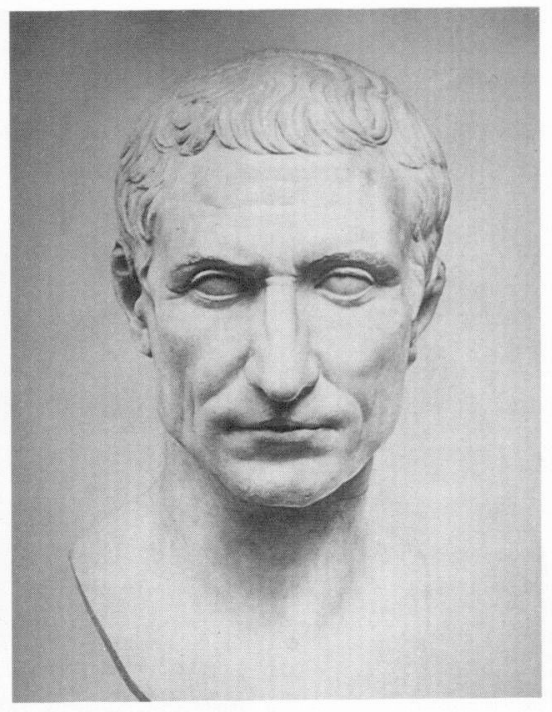

Abbildung 7

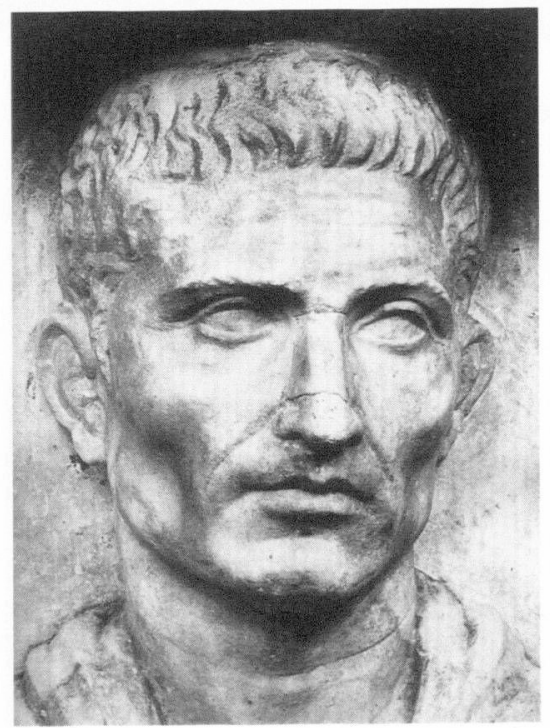

Abbildung 8

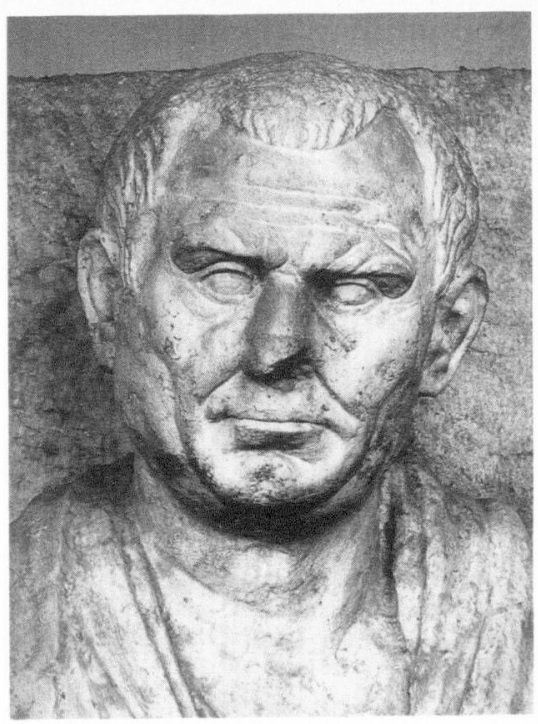

Abbildung 9 


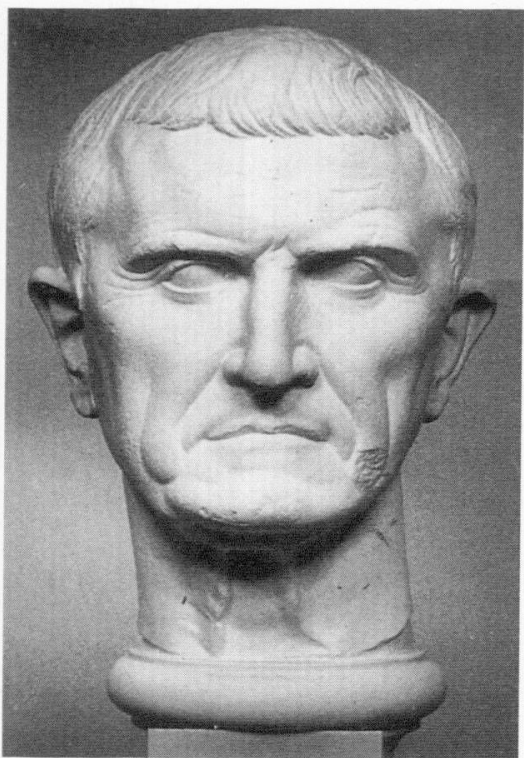

Abbildung 10

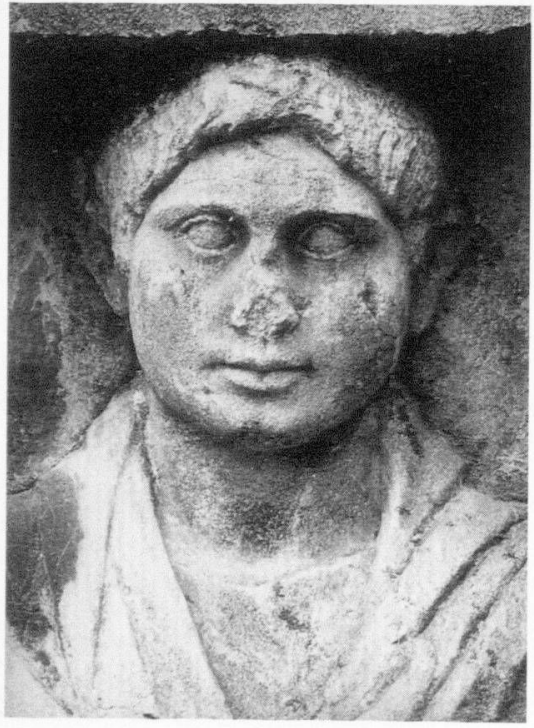

Abbildung 12

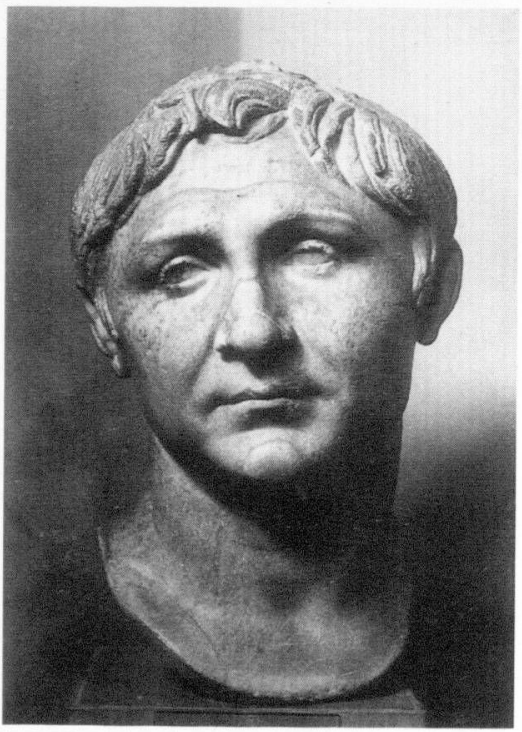

Abbildung 13 


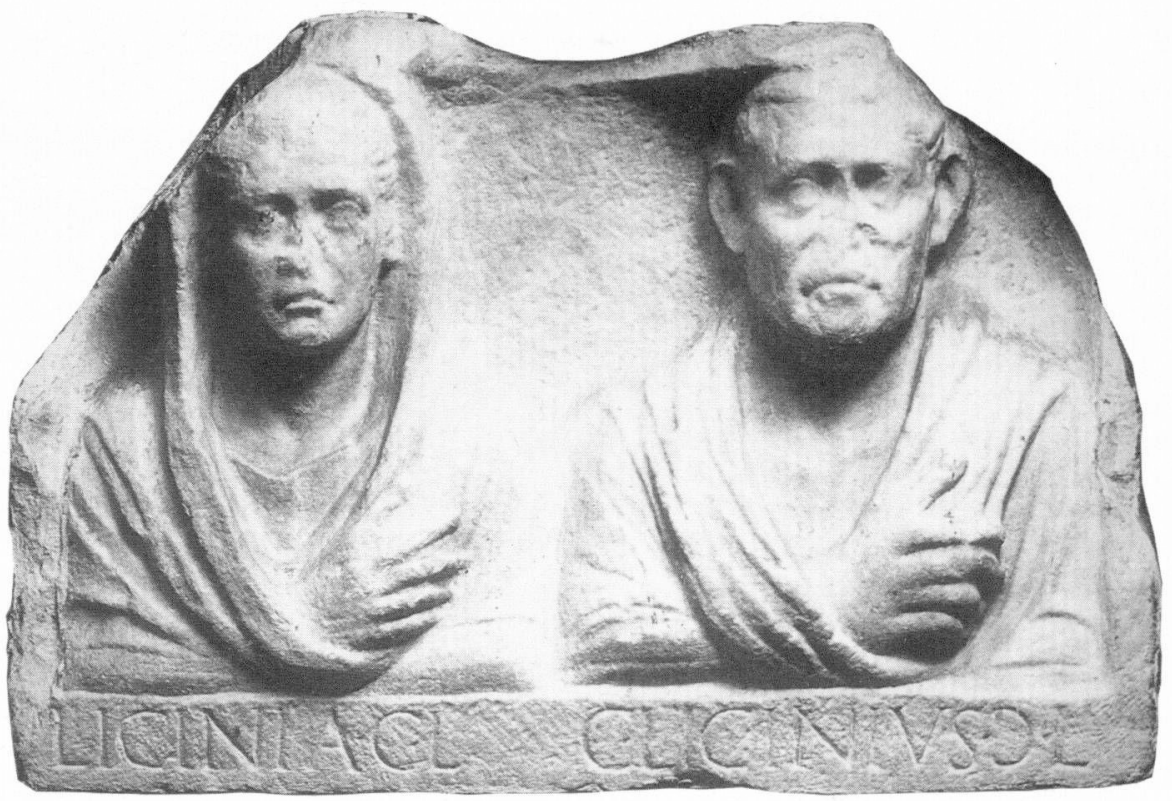

Abbildung 11 